Please send trade news information and illustrations to Arveen Bajaj at the $B D J$, Nature Publishing Group, The Macmillan Building, 4-6 Crinan Street, London N1 9XW. Trade news is provided as a service to readers using text and images from the manufacturer, supplier or distributor and does not imply endorsement by the $B D J$. Normal and prudent research should be exercised before purchase or use of any product mentioned.

\section{Micron accuracy}

The 'incise' process from Renishaw, specialists in industrial and CAD/CAM technologies, focuses on the accuracy of crown and bridge framework manufacture from start to finish.

The metal-free CAD/CAM system measures human error and the introduction of micron accuracy ensures correct restorations every time.

Chair time is reduced during final crown and bridge fitting appointments, while the restoration's resistance to tooth decay and staining is increased, resulting in a longer, lasting restoration.

The metal free benefits of the incise process eliminate the occasional problems when using metal crowns where oral tissues react with the restoration causing gum recession, exposing unattractive metal collars prone to tooth decay and staining. Renishaw's incise crowns and bridges are made with a biocompatible zirconia ceramic core which provides a strong framework, achieving excellent aesthetics, giving the look and function of natural teeth.

Reader response number 51

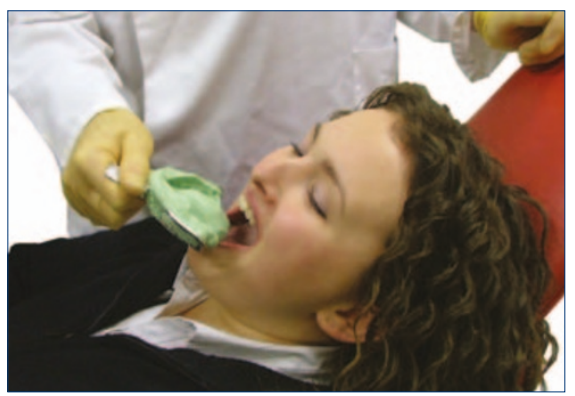

\section{Super-smooth finish}

Dentsply Ash Instruments has introduced a range of zirconium nitride coated restorative instruments to help get the best finish working with composite materials that can sometimes stick to the placement instrument.

The range includes over 20 instruments, on three handle choices, Lustra, Cearmicolor and Flexichange. Although the pale

\section{Antimicrobial agent}

Chlorhexidine Gluconate Medicated Mouthwash from Blackwell Supplies is the generic equivalent of Corsodyl. Chlorhexidine gluconate is a well-proven antimicrobial agent which reduces the formation of plaque and combats gingivitis and oral candida infections.

Chlorhexidine Gluconate Mouthwash is recommended for both pre- and postoperative use in the rapidly expanding areas of endodontics and dental implants, where leading dental specialists are increasingly exploiting its qualities to reduce infection and minimise patient discomfort. It is available in

\section{Treatment for periodontal pockets}

Chlo-Site from Oraldent, is a new, simple, and efficient, safe way to treat periodontal pockets. Chlo-Site is a gel with $1.5 \%$ chlorhexidine that is capable of assuring both active and passive disinfection coverage at the site where it was applied for up to two weeks, its maker claims.

Chlo-Site, to be used in conjunction with srp, remains microbiologically active for a minimum of 15 days with effective concentrations of two types of chlorhexidine, chlorhexidine digluconate - slow release $0.5 \%$ and chlorhexidine dihydrochloric - rapid release $1.0 \%$. This combination makes the bactericidal activity of Chlo-Site $40-60$

gold coloured zirconium nitride coating is so thin it adds no bulk to the instrument, it gives the instrument a super-hard, supersmooth finish.

The smooth finish means that the tip is very "non-stick", much more than the olderstyle anodised aluminium instruments. The coating hardness will also ensure the instrument does not become scratched, thereby staying smooth for longer

Reader response number 53

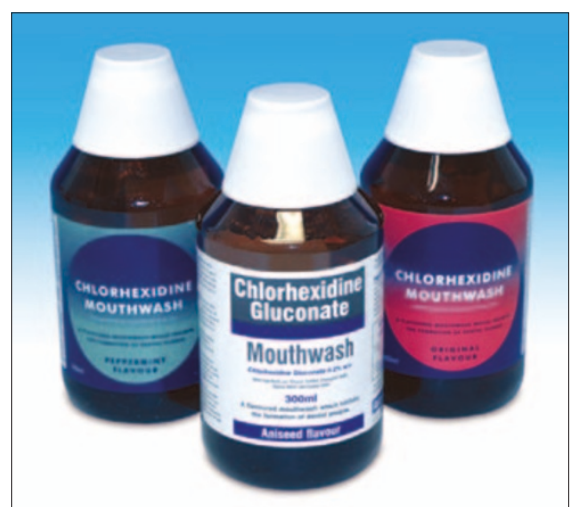

Original, Peppermint or Aniseed flavour to meet the needs of every patient.

Reader response number 50

times greater than the Minimum Inhibiting Concentration (MIC) for the pathogenic bacteria usually in periodontal pockets within the first week.

Its easy and ready to use gel is supplied in disposable syringes and can be applied directly into the periodontal pocket with the special periodontal needle provided for fast, accurate treatment of periodontal pockets.

Reader response number 52
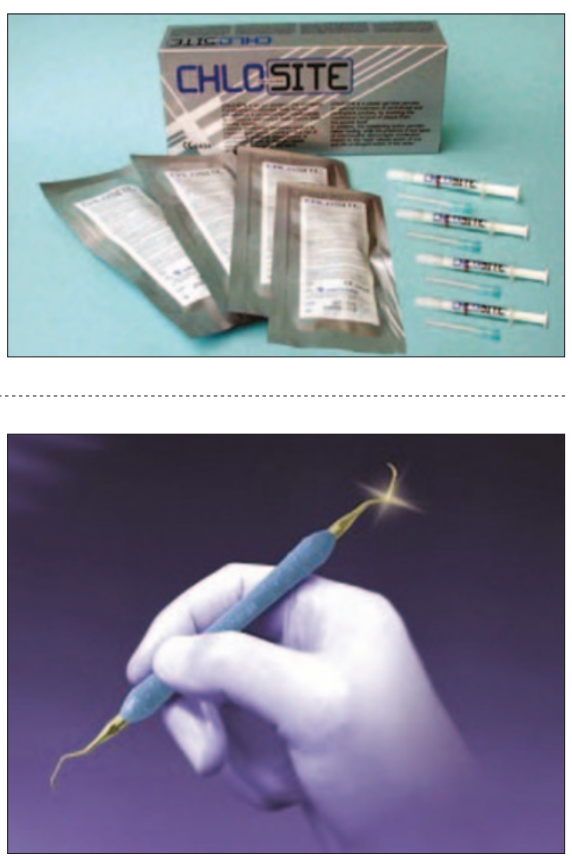


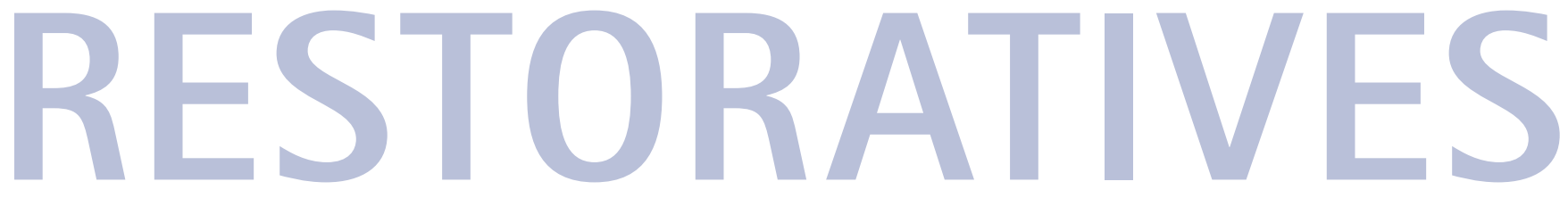

\section{Aesthetic resin cement}

Calibra, the aesthetic resin cement from Dentsply is designed to simplify your cementation needs for indirect cosmetic restorations. Calibra is simple-to-handle and is available as a paste system in five shades - translucent, light, medium, dark and opaque, providing a broad spectrum of shading possibilities.

Calibra's choice of catalyst paste viscosities means that you get the right handling for the right application. It can be used easily in all modes of curing light cure and dual cure and it sticks to your brush for easy lift-off.

The Calibra system, including Prime\&tBond NT dual cure, provides mechanical properties for long-lasting strength. The unique resin matrix in Calibra cement gives strength and aesthetics and it can be used for inlays/ onlays, veneers, crowns and bridges - and any cosmetic indirect restoration that demands aesthetics and strength.

Reader response number 55

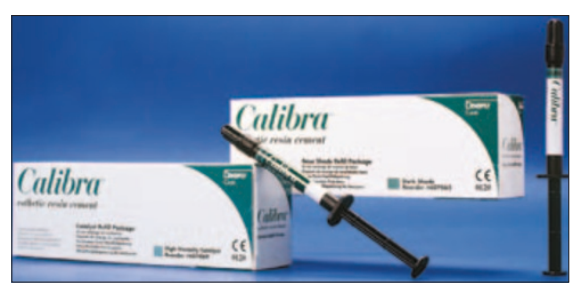

\section{Easy to use syringe}

Blackwell Supplies offers a wide range of practical solutions for the dental profession including the effective and easy to use Rotor Syringe range to its customers. Designed to eliminate the use of barbs, the Rotor S/A Syringe uses the elasticity of the cartridge and thumb disk to induce carefully and easily controlled aspiration.

\section{Translucency and aesthetics}

Fuji VIII is a glass ionomer cement with physical and aesthetic properties which can be used for Class III, V and root surface restorations. GC Fuji VIII GP

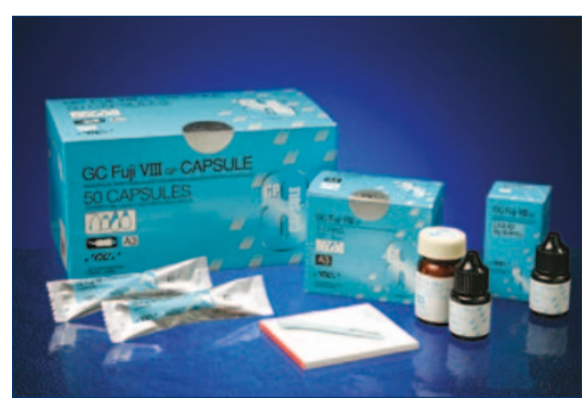

has been developed to provide the best translucency and a perfect match with Vita shades. The special formula incorporating patented resins provides better aesthetics, making it ideal for anterior restorations.

The product has good aesthetics, translucency without light curing, reliable bond strength to enamel and dentine, excellent physical properties, reaching $90 \%$ of its mechanical properties in ten minutes and is economical, says the company. Fuji VIII is available in either capsules or a hand mixed version.

Reader response number 54

\section{Non-stick formula}

New GC Gradia Direct Flo flowable composite can be used in conjunction with Gradia Direct and the shades can be matched to the colour concept of the easy to use, aesthetic composite.

Due to its physical properties and flow characteristics Gradia Direct Flo can be used as a liner, for blocking undercuts under composite restorations and for minimal intervention cavities.

The product's non-running and nonstick formula shapes to cavity walls and makes it easy to handle. This radiopaque material is supplied in a syringe design that allows for easy manipulation and

The Rotor Syringe can also be used with the Astra Self-Aspirating Cartridge. This method uses the unique and effective design of the Astra Cartridge to induce aspiration. Designed to be effective and practical, the Rotor Syringe is fully autoclavable, helping dentists to maintain the highest levels of hygiene and infection control within their surgeries.

The moulded components are made precise application. It is available in seven shades, A1, A2, A3, A3.5, A03, CV and BW.

Reader response number 56

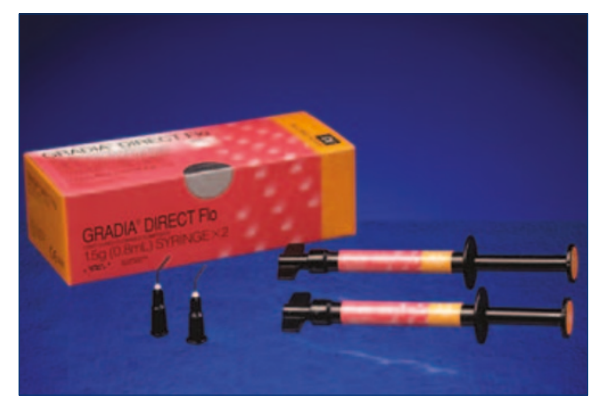

from PEEK, offering a lightweight well It also ensures that components will not rust after repeated exposure to autoclaving or chemicals commonly used within the dental surgery.

The Rotor Syringe is constructed from quality stainless steel, making it exceptionally comfortable to use.

Reader response number 57 balanced syringe, which is easy to use. 


\section{One step composite polishing}

The latest addition to the Shofu range includes One Gloss. It is a one-step finisher and polisher and can be used to fin-

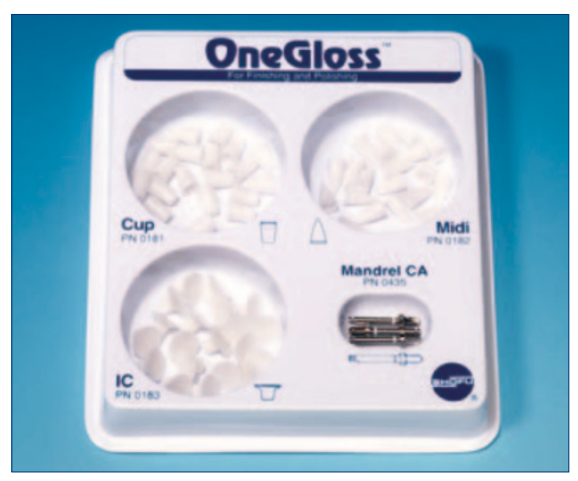

ish and polish all composite restorations simply by altering the contact pressure on the restoration, without having to change instrument.

One Gloss can also be used for the removal of stains or excess resin cement from tooth surfaces without damaging the enamel, which has proved to be particularly useful in orthodontic cases and polishing after scaling, thus reducing chairside time.

One Gloss is packaged in a set of 60 polishers (20 each of Cup, Midi Point and Inverted Cone shapes) and 3 x CA Mandrels plus instructions.

Reader response number $\mathbf{5 8}$

\section{Consistent mixing}

Dentsply's range of amalgams Dispersalloy and Megalloy EZ with ProMix amalgamator can cater to every alloy preference and technique, says the company.

ProMix provides a high degree of accuracy for more consistent mixing. The spring tensioned, moulded capsule holder enables ProMix to virtually adapt to every capsule design. The sealed mix area ensures that spills can be quickly and easily removed.

It has a sleek ergonomic design with touch pad controls and an LED display that is not only aesthetically pleasing in the office environment, but is also easy to load and unload. It also has a smooth

surface, compatible with many disinfectants.

The ProMix amalgamator with either Megalloy EZ capsules or Dispersalloy capsules can cater to your preferred technique.

Reader response number 60

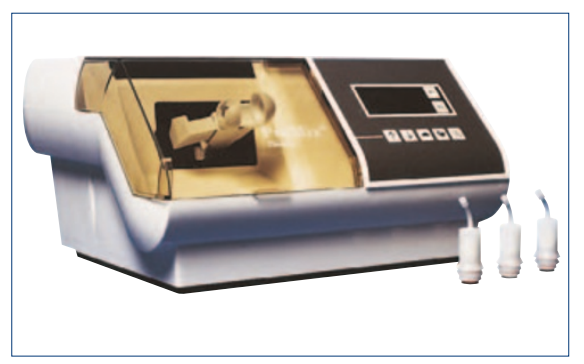

\section{For hard to reach areas}

NuBend $120^{\circ}$ is specially angled to allow injection of composite in hard to reach areas. It can be used in linguals of lower teeth, the distal of a terminal maxillary tooth and undercuts on crown preps that require coring.

With traditional ampules, instances occur where the composite injected simply pulls out when the ampule is removed. This necessitates placement of the material on an instrument and delivery to the awkward area, which causes air voids and compromised contours can result. NuBend $120^{\circ}$ enables easier access and direct injection of the composite.

In addition, Nubend composite is a light cured, resin based microhybrid allowing superior strength and polishability. The composite has a viscosity that is easy to handle and will not slump.

Reader response number 62

\section{Strength and beauty combined}

Procera Laminate withstands the rigors of both professional handling and patient use due to a $700 \mathrm{MPa}$ flexural strength. The layered NobelRondo porcelain ensures esthetic results and patient satisfaction, its makers claim.

The $0.25 \mathrm{~mm}$ Procera Laminate core of densely sintered aluminum oxide (alumina) is biocompatible, effectively masks underlying discoloration, and minimises staining issues during bonding. Also translucent, Procera Laminate preserves the tooth's luminescence.

Reader response number 59

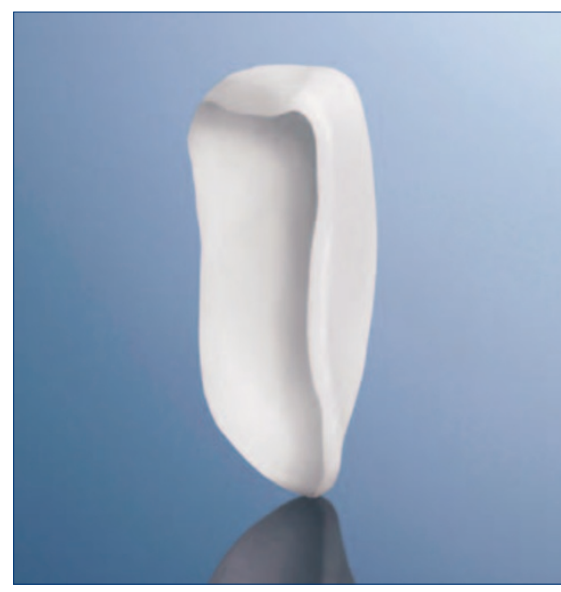

\section{Self-etching system}

OptiBond All-In-One from Kerr is a single-component, self-etch adhesive that eliminates multiple steps when bonding direct and indirect restorations. The light-cured adhesive provides superior adhesion to all surfaces and substrates, even indirect metal-based restorations. Its ternary solvent system provides enhanced shelf-life stability and effective enamel etching for long-term bond performance. OptiBond All-In-One SelfEtch Adhesive delivers penetration of dentin tubules, giving you exceptional bond strength and protection against microleakage and post-op sensitivity. Reader response number 61

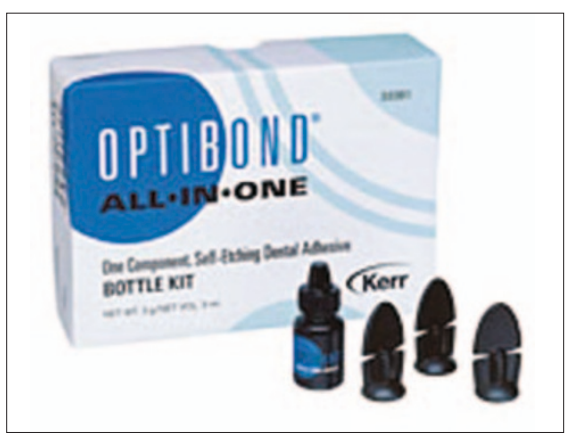

